\title{
Fatty acid composition of retail whole milk from Jersey and Guernsey cows over a two year study
}

S Stergiadis, CJ Seal, C Leifert, MD Eyre, G Butler

Newcastle University, Newcastle upon Tyne, United Kingdom

\author{
Email: socrates@nefg.net
}

Introduction The relatively high saturated fatty acid (SFA) content in milk fat has been linked with negative effects on human health. However, some monounsaturated fatty acids (MUFA), such as cis-9 C18:1 (oleic acid, OA) and trans-11 C18:1 (vaccenic acid, VA) and polyunsaturated fatty acids (PUFA) from the n-3 and n-6 series and cis-9trans-11 conjugated linoleic acid (CLA) found in milk, have been linked to beneficial effects on human health (Haug et al. 2007). C18:3n-3 (ALA) and C18:2n-6 (LA) are the main n-3 and n-6 fatty acids (FA) in milk, respectively. In other studies, milk collected from Jersey cows showed differences in the FA profile compared with milk from Holstein cows (Carroll, 2006) which are almost exclusively used in the UK. Seasonal effects, comparing summer diets with high intakes of fresh grass, with winter diets based on silages and concentrates, have been found to increase the concentrations of ALA, VA, CLA and PUFA in milk (Chilliard et al., 2000). Milk collected in this survey was derived from conventional farms, in order to avoid the effects of organic management on milk FA profile, previously shown in retail milk (Stergiadis, 2009).The aim of this study was to investigate the FA composition of retail whole milk labelled as "Jersey" or "Jersey \& Guernsey" during summer and winter periods to establish whether the differences reported in previous studies at the individual cow level were also seen in commercial milk.

Materials and methods In this survey, 16 different sources of commercial full-fat milk, characterised by brand and animal breed, were collected from retail outlets in the North East of England. 4 brands were labelled as "Jersey" or "Jersey and Guernsey" milk (JG) while the other 12 sources indicated no specification about the animal breed used (NS). Milk purchase was carried out over two years on 4 sampling dates: 2 during winter (January 2007/2008) and 2 during summer (August 2006/2007). Analysis of FA methyl esters was performed with a Gas Chromatography system (Shimadzu, GC-2014, Japan) using a Varian CP-SIL 88 fused silica capillary column (100m x $0.25 \mathrm{mmID}$ x $0.2 \mu \mathrm{m}$ film thickness). Peaks were identified using a 39 FAME and CLA standards. Analysis of variance (ANOVA) using linear mixed effects model was used to analyze results in R statistical environment using "year" ( $1^{\text {st }}$ or $\left.2^{\text {nd }}\right)$, "season" (winter or summer) and "breed" (JG or NS) as fixed factors and milk ID as random factor.

Results Table 1 shows that there was no effect of breed on the individual PUFA concentrations in milk, while JG milk had lower concentrations of OA than NS milk. In contrast, the seasonal influence was high and summer milk was associated with higher concentrations of OA, VA, CLA and ALAcompared with winter milk. Table 2 shows that JG milk had higher concentrations of SFA, lower concentrations of MUFA and tended to show lower concentrations of PUFA than NS milk. In addition, summer milk had higher concentrations of MUFA, PUFA and n-3 FA and lower concentrations of SFA than winter milk. Milk LA concentrations were not affected by either season or animal breed. Fat content of milk expressed as $g$ fat $/ 100 \mathrm{~g}$ milk was; $4.80 \%$ for JG milk, $3.49 \%$ for NS milk, 3.73\% for summer milk and 3.92\% for winter milk with the differences between JG vs NS and summer vs winter being statistically significant. individual fatty acids in JG and summer milk compared with NS and winter milk

\begin{tabular}{lllll}
\hline \hline & JG & P-value & Summer & P-value \\
\cline { 2 - 5 } OA & $-10.4 \%$ & $* *$ & $+14.2 \%$ & $* * *$ \\
VA & $+14.3 \%$ & ns & $+109.8 \%$ & $* * *$ \\
CLA & $-7.4 \%$ & ns & $+70.3 \%$ & $* * *$ \\
ALA & $-1.0 \%$ & ns & $+38.2 \%$ & $* * *$ \\
LA & $-8.5 \%$ & ns & $-2.8 \%$ & ns \\
\hline
\end{tabular}

Significances were declared at $* * *: \mathrm{P}<0.001, * *: \mathrm{P}<0.01, \uparrow: 0.05<\mathrm{P}<0.10$, ns: $\mathrm{P}>0.10$
Table 1 Differences in relative proportions (\%) of

Table 2 Differences in relative proportions (\%) of fatty acid groups in $\mathrm{JG}$ and summer milk compared with NS and winter milk

\begin{tabular}{lllll}
\hline \hline & JG & P-value & Summer & P-value \\
\cline { 2 - 5 } SFA & $+3.8 \%$ & $* *$ & $-5.8 \%$ & $* * *$ \\
MUFA & $-9.5 \%$ & $* *$ & $+16.2 \%$ & $* * *$ \\
PUFA & $-7.6 \%$ & $\dagger$ & $+13.4 \%$ & $* * *$ \\
n-3 & $+0.4 \%$ & ns & $+31.9 \%$ & $* * *$ \\
n-6 & $-9.8 \%$ & ns & $-2.2 \%$ & ns \\
\hline
\end{tabular}

Conclusions Retail milk without breed specification showed a slightly better fatty acid profile than retail milk from Jersey \& Guernsey cows. The relatively high content of SFA shown in other studies for Jersey milk has been confirmed for milk at the consumer level although the beneficial PUFA concentrations were not different between the different sources of milk. The concentrations of the FA groups and individual FA that have previously been associated with positive effects in human health were higher in summer milk compared with winter milk. This also raises the importance for improvement of the FA profile of winter milk, possibly by the nutritional management of cows.

Ackowledgements The authors gratefully acknowledge financial support from the European Community under the 6th Framework Programme Integrated Project "QualityLowInputFood", FP6-FOOD-CT-2003-506358, the Greek State Scholarship Foundation and the Yorkshire Agricultural Society.

\section{References}

Carroll, S.M., DePeters, E.J., Taylor, S.J., Rosenberg, M., Perez-Monti, H.and V.A. Capps. 2006. Animal Feed Science and Technology 131, 451-473

Chilliard, Y., Ferlay, A., Mansbridge, R.M. and M. Doreau. 2000. Ann Zootech 49,181-205.

Haug, A., Hostmark, A.T., and O.M. Harstad. 2007 Lipids Health Dis 6, 25

Stergiadis, S., Seal., C.J., Leifert, C., and G. Butler. 2009 Proceedings of BSAS, 88 